\title{
SASIS collecte des données sans bases contractuelles
}

\section{Ernst Gähler ${ }^{a}$, \\ Thomas Kessler ${ }^{b}$, \\ Gabriela Langc}

a Dr en médecine, vice-président de la FMH, responsable du domaine Tarifs et Conventions

b Chef de projet, domaine Tarifs et Conventions de la $\mathrm{FMH}$

c Service juridique de la FMH

\section{SASIS: comportement non conforme \\ à la convention}

Selon la convention-cadre TARMED, tout médecin souhaitant ouvrir un cabinet doit disposer d'un numéro de registre des codes créanciers, appelé aussi numéro RCC. Ces numéros sont délivrés par l'entreprise SASIS SA, mandatée par santésuisse, et ce pour toute la Suisse. Pour obtenir un numéro RCC, le médecin doit fournir un grand nombre de documents dont une autorisation cantonale d'exercer, ses attestations de formation complémentaire de la FMH ainsi que ses titres de formation approfondie de la FMH. En outre, il doit remplir un questionnaire détaillé [1].

Ce questionnaire de trois pages comporte différentes catégories. Les données des catégories «Données de base», «Nom et adresse», «Données des paiements» et «Admission» sont nécessaires pour pouvoir examiner le droit de facturer du médecin. Les catégories «Formation et qualifications», «Statut», «Orientation médicale», «Installation et équipement» et «Rapport» contiennent quant à elles en grande partie des questions et des données qui ne sont pas utiles pour la facturation.

Enfin, le médecin doit confirmer par sa signature qu'il a pris note que «Toute information fausse ou incomplète ainsi que la non-communication des mutations peuvent conduire au retrait du numéro RCC ou du numéro C».

\section{«La FMH s'engage sur le plan politique en faveur d'une nouvelle pratique en matière d'évaluation de l'économicité.»}

santésuisse justifie sa soif d'information en affirmant que ces données sont nécessaires «pour classer [le médecin] dans le groupe de médecins adéquat en vue de l'examen de l'économicité». Or la FMH sait, sur la base des centaines de cas qu'elle a rencontrés, à quel point les examens d'économicité (fondés sur la méthode ANOVA) menés par santésuisse peuvent manquer de transparence, se révéler incomplets et partiellement insuffisants d'un point de vue statis- tique. C'est d'ailleurs ce qu'ont récemment confirmé deux arrêts du Tribunal fédéral [2]. Sur ce point aussi, la FMH s'engage sur le plan politique en faveur d'une nouvelle pratique en matière d'évaluation de l'économicité.

Le questionnaire SASIS exige des médecins qu'ils fournissent des données qui vont, selon la FMH, bien au-delà des conventions TARMED, et qui ne sont dès lors pas fondées sur des bases légales. C'est pourquoi nous allons examiner les questionnaires au cours des semaines à venir et informer prochainement les médecins de la façon de se défendre contre une telle soif d'information.

\section{Le numéro $C$ n'est pas indispensable!}

Certains médecins et personnes appartenant à d'autres groupes de professions, dont par exemple les psychothérapeutes salariés (qui fournissent des prestations médicales déléguées dans le cadre du TARMED), nous ont rapporté que santésuisse, invoquant l'art. 36a LAMal, a exigé d'eux, en tant que salariés d'une institution, qu'ils demandent un numéro de contrôle (payant), appelé aussi numéro C.

Selon la convention-cadre TARMED et les conventions cantonales d'adhésion, la facture adressée aux assureurs doit comporter à la fois le numéro GLN (Global Location Number, anciennement numéro EAN) et le numéro RCC. Les conventions ne prévoient cependant aucune indication d'un numéro $\mathrm{C}$ pour les médecins salariés et les psychothérapeutes.

Le Tribunal fédéral est clair [3]: si santésuisse a besoin de davantage de données que celles prévues par les conventions, «les conventions [doivent] être complétées de façon à tenir compte des modalités de facturation de cette catégorie de prestataires». Jusqu'ici, les partenaires tarifaires santésuisse et la FMH n'ont jamais convenu que le numéro $\mathrm{C}$ soit indiqué sur la facture. santésuisse ne peut par conséquent obliger ni les médecins ni les personnes appartenant à d'autres groupes de professions et qui fournissent des prestations dans le cadre du TARMED, à demander un numéro C.

Depuis des années, la FMH tient quotidiennement à jour la banque de données www.doctorfmh.ch, et ce dans le respect des conventions et des mandats, et en y consacrant beaucoup de temps. Cette banque de données accessible à tous présente de manière trans- 


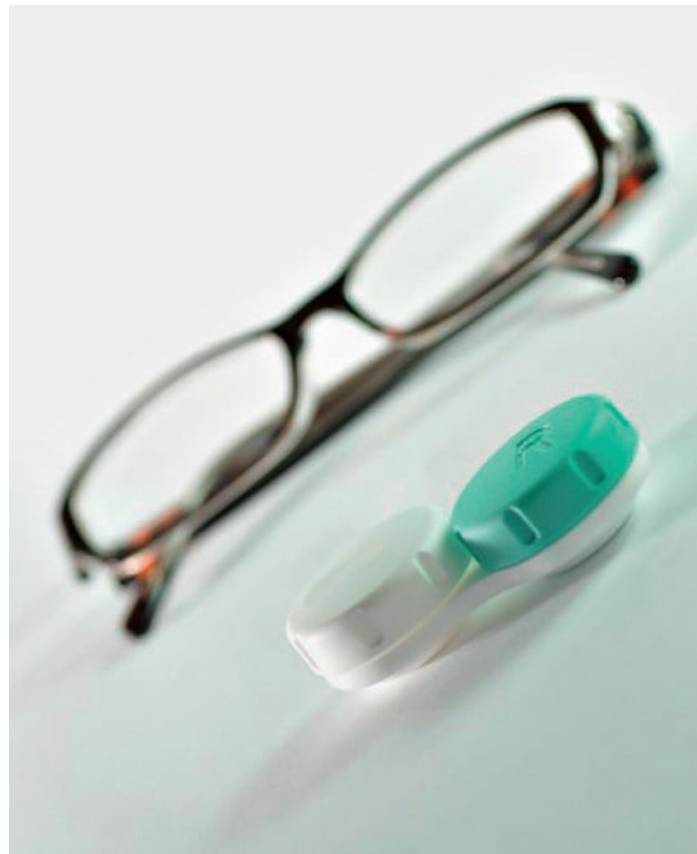

Verres de lunettes et lentilles de contact seront supprimés de la Liste des moyens et appareils (LiMA).

parente les qualifications professionnelles de tous les médecins de Suisse.

\section{La modification de la LiMA a peu d'effets sur la facturation du matériel à usage courant selon le TARMED}

Les éléments suivants seront supprimés de la Liste des moyens et appareils (LiMA) au $1^{\text {er }}$ juillet 2011:

- 03.05.10.00.1 Gripper pour Port-A-Cath

- 03.05.11.00.1 Aiguille pour Port-A-Cath

- 25.01 Verres de lunettes/lentilles de contact

- 34.50 Plâtres et accessoires pour plâtres

- 34.90 Système de traitement des plaies par pression négative
La modification de la Liste des moyens et appareils (LiMA) a peu d'effets sur la facturation du «matériel à usage courant» dans le cadre des prestations médicales et non médicales selon le TARMED. Indépendamment du fait que le «matériel à usage courant» soit listé ou non dans la LiMA, les interprétations générales IG-20 Matériel à usage courant et implants inscrites dans le TARMED restent valables: «Le matériel à usage courant peut être facturé séparément, pour autant que le prix d'achat dépasse 3 CHF par pièce (TVA comprise). Est à facturer, le prix coûtant (c'est-à-dire le prix unitaire découlant de la quantité annuelle) auquel vient s'ajouter une majoration de $10 \%$. Pour le matériel à usage courant figurant dans la LiMA ou dans des conventions, le prix indiqué ne peut être dépassé.

Chaque article doit être mentionné avec son prix et la date de la remise (date de la séance).

Les instruments réutilisables (y compris les fixateurs externes) ne sont pas concernés par ces dispositions d'indemnisation. Ils sont déjà pris en compte dans les diverses positions tarifaires.

Les confections sur mesure par les techniciens orthopédistes ou les bottiers orthopédistes ne font pas non plus partie de ces dispositions d'indemnisation. De telles confections particulières sont indemnisées selon les tarifs correspondants (tarif de l'Association suisse des techniciens orthopédistes (ASTO) ou tarif de l'Association suisse pour la technique orthopédique de chaussures (OSM).»

\section{Références}

1 cf. http://www.santesuisse.ch/user_content/editor/ files/SASIS_ZSR_Fragebogen_10-01-06/questionnaire_ medecin.pdf

2 BGE 9C_968/2009 du 15 décembre 2010, BGE 9C_733/2010 du 19 janvier 2011.

3 BGE 9C_701/2008 du 20 avril 2009. 